\title{
ON A PROBLEM OF ORE ON MAXIMAL TREES
}

\author{
S. B. RAO \\ (Received 18 March 1969) \\ Communicated by E. Strzelecki
}

We consider only graphs without loops or multiple edges. Pertinent definitions are given below. For notation and other definitions we generally follow Ore [1].

A connected graph $G=(X, E)$ is said to have the property $P$ if for every maximal tree $T$ of $G$ there exists a vertex $a_{T}$ of $G$ such that distance between $a_{T}$ and $x$ is same in $T$ as in $G$ for every $x$ in $X$. The following problem has been posed by Ore (see [1] page 103, problem 4): Determine the graphs with property $P$. This paper presents a solution to the above problem in the finite case.

THEOREM 1: A finite biconnected graph $G=(X, E)$ has the property $P$ if and only if it is a cycle (type $I)$ or a complete bipartite graph $K(V, X-V)$ with $|V|=2$ and $|X-V| \geqq 2$ (type $I I$ ).

Proof. It is easy to check that the graphs mentioned in the statement of the theorem have the property $P$.

Conversely, let $G$ be a finite biconnected graph with property $P$ and $d(G)$ its diameter. If $d(G)=1$, then, $G$ is'a triangle. So assume that $d(G) \geqq 2$. We note the following facts.

(1) If $T$ is a maximal tree of $G$ then $d(T) \leqq 2 d(G)$ and further if $d(T)=2 d(G)$ then $a_{T}$ (given by the property $P$ ) is the unique centre of $T$.

(2) Every subgraph of $G$ which is a tree can be extended to a maximal tree of $G$.

Let $x_{0}, y_{0}$ be vertices of $G$ such that $d_{G}\left(x_{0}, y_{0}\right)=d(G)$. Since $G$ is biconnected there is a simple circuit $\mu$ containing $x_{0}, y_{0}$ (Theorem 5.4.3 of [1]). Without loss of generality assume that $\mu=\left[x_{0}, x_{1}, x_{2}, \cdots, x_{t}, x_{0}\right]$. Clearly the length of $\mu, L(\mu)$ is greater than or equal to $2 d(G)$. We show that $L(\mu) \leqq 2 d(G)+1$. Suppose not, then consider the subgraph $\mu\left[x_{0}, x_{t}\right]$ whose length $\geqq 2 d(G)+1$. By (2) and (1) we get a contradiction.

CASE (i). $L(\mu)=2 d(G)+1$. Let $A=\left\{x_{0}, x_{1}, \cdots, x_{i}\right\}$. Then $X=A$. For otherwise let $y$ be a vertex of $X-A$ adjacent to some vertex of $A$, say $x_{i}$. Consider the subgraph $\xi=\left(y, x_{i}\right)+\mu\left[x_{i}, x_{0}\right]+\mu\left[x_{0}, x_{i-1}\right]$ whose diameter $\geqq 2 d(G)+1$. By (2) and (1) we get a contradiction. 
Now $G=\mu$. Otherwise, let $\left(x_{i}, x_{j}\right)$ be an edge of $G$, where $j$ is different from $i-1$ and $i+1$. Consider the subgraph $T=\mu\left[x_{i+1}, x_{j}\right]+\left(x_{j}, x_{i}\right)+\mu\left[x_{0}, x_{i-1}\right] . T$ is a maximal tree of $G$ and $d(T)=2 d(G)$. Since $G$ has the property $P$, by (2), $a_{T}$ is the unique centre of $T$, but here it is not, a contradiction. Hence $G$ is a cycle (Type 1).

CASE (ii). $L(\mu)=2 d(G)$. Let $A=\left\{x_{0}, x_{1}, \cdots, x_{t}\right\}$. Define $B_{i}=\{y: y \in X$ $-A$ and $y$ is adjacent to $x_{i}$ in $\left.G\right\}$, for every $i, 0 \leqq i \leqq t$ and $B=\bigcup_{i=0}^{t} B_{i}$. If $B$ is empty $G=\mu$ (as in case (i)). Assume that $B$ is non empty. We show that $B$ is an independent set in $G$. Let if possible $x, y$ be vertices in $B$ and $(x, y)$ be an edge of $G$ with $y$ in $B_{i_{0}}$. Then consider the following subgraph

$$
\xi=(x, y)+\mu\left[x_{i_{0}}, x_{0}\right]+\mu\left[x_{0}, x_{i_{0}-1}\right]
$$

of $G$ whose length is $2 d(G)+1$; by (2) and (1) this leads to a contradiction. Further, if $z$ is in $B_{i},\left(z, x_{i+1}\right),\left(z, x_{i-1}\right)$ are not edges of $G$. Since $B$ is an independent set and $G$ is biconnected, $z$ is joined to $x_{j}$ for some $j, 0 \leqq j \leqq t$ and $i \notin\{i-1, i+1\}$. If $d(G)>2$ consider the subgraph

$$
\xi=\left[x_{j+1}, x_{j+2}, \cdots, x_{i}, z, x_{j}, x_{j-1}, \cdots, x_{i+1}\right] .
$$

By (2) this can be extended to a maximal tree $T$ of $G$ and $d(T)=2 d(G)$ but $a_{T}$ is not the unique centre of $T$-a contradiction. Hence $d(G)=2$ so $\mu=\left[x_{0}, x_{1}, x_{2}, x_{3}, x_{0}\right]$. Since $B$ is nonempty at least one of $B_{i}, 0 \leqq i \leqq 3$ is nonempty. Assume that $B_{0}$ is non empty. Now if $x$ is in $X-A$ it belongs to $B_{0}$ and $B_{2}$. Let $V=\left\{x_{0}, x_{2}\right\}$ then $G=K(V, X-V)$, the complete bipartite graph, with $|X-V| \geqq 2$ (type II). This completes the proof of theorem 1 .

THEOREM 2. A finite connected graph with property $P$ on $n$ vertices is a tree or consists of a subgraph $H$ on $n_{0}\left(3 \leqq n_{0} \leqq n\right)$ vertices of type $I$ or type II to which trees with a total of $n-n_{0}$ edges are attached at some vertices of $H$.

Proof. Let $x$ be a cut vertex of $G$. It can be easily shown that at most one leaf with respect to $x$ of $G$ is not a tree. Now theorem 2 follows from theorem 1 .

Remark. Perhaps it is true that $G=K(V, X-V)$, the complete bipartite graph with $|V|=2$, is the only biconnected graph with property $P$ if $X$ is infinite.

\section{Reference}

[1] O. Ore, Theory of graphs (American Mathematical Society Colloquium Publication, 38, Providence, Rhode Island, 1962).

Indian Statistical Institute

Calcutta 35

India 